\title{
BMJ Open DNA sequence analysis and genotype- phenotype assessment in 71 patients with syndromic hearing loss or auditory neuropathy
}

\author{
Hsiao-Yuan Tang, ${ }^{1}$ Ping Fang, ${ }^{2,3}$ Jerry W Lin, ${ }^{1}$ Sandra Darilek, ${ }^{3}$ \\ Brooke T Osborne, ${ }^{1}$ Jo Ann Haymond, ${ }^{1}$ Spiros Manolidis, ${ }^{4}$ Benjamin B Roa, ${ }^{5}$ \\ John S Oghalai, ${ }^{6}$ Raye L Alford ${ }^{1}$
}

To cite: Tang $\mathrm{H}-\mathrm{Y}$, Fang $\mathrm{P}$, Lin JW, et al. DNA sequence analysis and genotypephenotype assessment in 71 patients with syndromic hearing loss or auditory neuropathy. BMJ Open 2015;5:e007506. doi:10.1136/bmjopen-2014007506

- Prepublication history for this paper is available online. To view these files please visit the journal online (http://dx.doi.org/10.1136/ bmjopen-2014-007506).

Received 22 December 2014 Revised 23 March 2015 Accepted 10 April 2015

CrossMark

For numbered affiliations see end of article.

Correspondence to Dr Raye L Alford; ralford@bcm.edu.

\section{ABSTRACT}

Objectives: Aetiological assessment of 71 probands whose clinical presentation suggested a genetic syndrome or auditory neuropathy.

Methods: Sanger sequencing was performed on DNA isolated from peripheral blood or lymphoblastoid cell lines. Genes were selected for sequencing based on each patient's clinical presentation and suspected diagnosis. Observed DNA sequence variations were assessed for pathogenicity by review of the scientific literature, and mutation and polymorphism databases, through the use of in silico tools including sorting intolerant from tolerant (SIFT) and polymorphism phenotyping (PolyPhen), and according to the recommendations of the American College of Medical Genetics and Genomics for the interpretation of DNA sequence variations. Novel DNA sequence variations were sought in controls.

Results: DNA sequencing of the coding and nearcoding regions of genes relevant to each patient's clinical presentation revealed 37 sequence variations of known or uncertain pathogenicity in 9 genes from 25 patients. 14 novel sequence variations were discovered. Assessment of phenotypes revealed notable findings in 9 patients.

Conclusions: DNA sequencing in patients whose clinical presentation suggested a genetic syndrome or auditory neuropathy provided opportunities for aetiological assessment and more precise genetic counselling of patients and families. The failure to identify a genetic aetiology in many patients in this study highlights the extreme heterogeneity of genetic hearing loss, the incompleteness of current knowledge of aetiologies of hearing loss, and the limitations of conventional DNA sequencing strategies that evaluate only coding and near-coding segments of genes.

\section{BACKGROUND}

Genetic hearing loss demonstrates extreme locus and allelic heterogeneity. ${ }^{1-4}$ More than 400 genetic syndromes include hearing loss

\section{Strengths and limitations of this study}

- As a research study, it was possible to perform DNA sequencing of a greater number of genes for each patient than would have been economically feasible by clinical genetic testing.

- Patients were followed over time allowing ongoing assessment of phenotypes and hearing status, and continuous refinement of suspected aetiologies.

- Patients were evaluated using a multidisciplinary team approach that included otolaryngologists, clinical geneticists, audiologists, speech and language therapists, and others, as appropriate for each patient, thus enhancing phenotypic assessment.

- The small number of patients evaluated in this study limits the number of genetic variants identified.

- This study was not designed to order clinical diagnostic assessments solely for research purposes; as such, assessment of phenotypes and estimations of potential aetiologies for hearing loss are limited to what was observed by physicians in the course of routine clinical care for patients with hearing loss.

as a feature, and more than 100 genes and genetic loci have been associated with nonsyndromic genetic hearing loss. Diagnosis of many syndromic forms of hearing loss can be made based on physical findings, while diagnosis of many others, especially syndromes with variable, non-specific or age-related features, is facilitated by genetic testing. Additionally, many causes of non-syndromic genetic hearing loss demonstrate similar audiometric profiles. Aetiological assessment of non-syndromic genetic hearing loss is greatly aided by genetic testing. ${ }^{1-4}$

Distinct physical findings associated with many syndromic forms of hearing loss direct 
targeted DNA sequence analysis towards particular genes. For example, enlarged vestibular aqueducts suggest Pendred syndrome and mutations in SLC26A4. Retinitis pigmentosa suggests Usher syndrome and mutations in MYO7A, USH1C, CDH23, USH2A or other Usher syndrome-associated genes. Pigmentary anomalies suggest Waardenburg syndrome and mutations in $P A X 3$, MITF, SOX10 or other Waardenburg syndrome-associated genes. Prolonged QT interval suggests Jervell and Lange-Nielsen syndrome and mutations in KCNQ1 or KCNE1. Clinical features of Cornelia de Lange syndrome suggest mutations in NIPBL, SMC1A or SMC $3 .^{1-4}$ Numerous other syndrome-gene associations have also been described. ${ }^{1-4}$

Auditory neuropathy is a distinct form of hearing loss where the outer hair cells function appropriately but sound is not transmitted properly to the brain. Although auditory neuropathy may occur as part of a syndrome, it may also occur as an isolated finding associated with mutations in OTOF, PJVK or DIAPH $3 .^{1-5}$

In this study, DNA sequencing was performed for 71 probands with hearing loss, whose clinical presentation suggested a genetic syndrome or auditory neuropathy. Sequencing of the coding and near-coding regions of genes relevant to each patient's clinical presentation revealed 37 sequence variations of known or uncertain pathogenicity in 25 patients. Fourteen novel sequence variations were discovered. Assessment of phenotypes revealed notable findings in nine patients.

\section{METHODS}

\section{Patients}

Patients with hearing loss of suspected genetic aetiology were identified through the clinical care centres of Baylor College of Medicine and Texas Children's Hospital. Parents of patients were offered enrolment in this study, where appropriate, to clarify their children's genetic test results. Written informed consent was obtained from all study participants or, in the case of minor children, at least one parent or legal guardian. Clinical evaluations of patients were conducted by physicians in accordance with routine clinical care for patients with hearing loss and the physicians' best clinical judgement. ${ }^{6}$

\section{Controls}

Controls were obtained from the Baylor Polymorphism Resource of Baylor College of Medicine. The control group consisted of $\geq 50$ individuals from each of four ancestral groups: African-American, Asian, Caucasian and Hispanic.

\section{Specimen collection and DNA isolation}

Blood was collected by peripheral venipuncture for the purposes of DNA isolation and the establishment of lymphoblastoid cell lines. Lymphoblastoid cell lines were established by standard Epstein-Barr virus-mediated transformation. DNA was isolated from blood samples and cell lines using PUREGENE DNA Purification Kits (Qiagen, Valencia, California, USA) for whole blood or cultured cells according to the manufacturer's specifications.

\section{DNA sequencing}

Clinical or research-based DNA sequencing of GJB2 was performed for all patients in this study group. Additional genes were selected for sequence analysis based on clinical findings. PCR amplification, and Sanger sequencing of the coding and near-coding regions of selected genes, were performed according to standard protocols. Primer sequences, and PCR and sequencing conditions, will be provided on request.

\section{Nomenclature}

DNA and protein sequence variations are named according to standard nomenclature recommendations. ${ }^{7}$

\section{Interpretation of DNA sequence variations}

Observed DNA sequence variations were assessed for pathogenicity by review of the scientific literature, and mutation and polymorphism databases,${ }^{8-11}$ through the use of in silico tools including sorting intolerant from tolerant (SIFT) and polymorphism phenotyping (PolyPhen), ${ }^{12} 13$ and according to the recommendations of the American College of Medical Genetics and Genomics for the interpretation of DNA sequence variations. ${ }^{14}$ The pathogenicity of previously reported DNA sequence variations was interpreted on the preponderance of evidence from prior reports and the predicted effect on the encoded protein product. Novel DNA sequence variations were interpreted as pathogenic mutations if they predicted nonsense codons or frameshifts followed by nonsense codons, occurred within splice site consensus sequences, were de novo changes in autosomal dominant conditions, or occurred at a position where a different nucleotide substitution had previously been reported as pathogenic. Novel DNA sequence variations were interpreted as variants of uncertain pathogenicity (VUS) if they predicted missense or synonymous codons or occurred near but not within canonical splice site consensus sequences. Novel DNA sequence variations identified in patients were sought in controls.

\section{RESULTS}

Sixty-seven probands with hearing loss were diagnosed with or suspected of having a genetic syndrome based on clinical observations: 47 patients had an enlarged vestibular aqueduct, Mondini malformation or other inner ear malformations; 7 patients had Usher syndrome-2 with type 1,5 with type 2; 1 patient had enlarged vestibular aqueducts and Usher syndrome type 2; 6 patients had Waardenburg syndrome-3 with type 1 , 1 with type 4,2 with peripheral demyelinating neuropathy, central 
dysmyelination, Waardenburg syndrome, Hirschsprung disease $(\mathrm{PCWH}) ; 5$ patients had prolonged QT interval1 with signs of VACTERL association (vertebral defects, anal atresia, cardiac defects, tracheo-oesophageal fistula, renal anomalies, limb defects); and 1 patient had Cornelia de Lange syndrome. Four probands with auditory neuropathy were also included in this study group: three had no known additional relevant phenotypic findings; one had brachycephaly, asymmetric facies and cupped ears.

All patients had clinical or research-based sequencing of GJB2, which excluded GJB2 as the cause of their hearing loss. Additional genes for DNA sequence analysis were selected for each patient based on clinical findings. Among the 71 patients in this study group, 37 different DNA sequence variations of known or uncertain pathogenicity were observed in the coding and near-coding regions of relevant genes in 25 patients, including 1 regulatory, 1 translation start site, 18 missense, 3 nonsense, 1 synonymous, 7 splice site and 6 frameshift mutations. Fourteen of the observed variants were understood to be novel at the time this manuscript was written, including five missense, two nonsense, one synonymous, four splice site and two frameshift mutations. Of these 14 novel variants, 7 were interpreted as mutations ( 1 de novo missense, 2 nonsense, 2 splice site, 2 frameshift) and 7 were deemed to be of uncertain pathogenicity (4 missense, 1 synonymous, 2 splice site; table 1). Only one of the novel variants discovered in this study was observed in $>400$ control chromosomes, which included at least 100 chromosomes each of African-American, Asian, Caucasian and Hispanic ancestry: the USH2A p.Thr3976Thr (c.11928G >A) variant was observed in 1 of 106 control chromosomes of Caucasian ancestry.

As shown in table 1, two patients carried apparently homozygous DNA sequence variations. The parents of the patient with an apparently homozygous mutation in USH1C denied consanguinity but are from the same small village. The parents of the patient with three apparently homozygous VUS in $\mathrm{CDH} 23$ are first cousins (table 1).

With the exception of the patient shown in table 1 to carry one mutation and three VUS in OTOF, benign polymorphisms were not included in this report. An exception was made for the presumptive p.Glu801Leu polymorphism, however, because the phase for this two nucleotide substitution could not be set in this patient, that is, $G A>T T$ in cis versus $G>T$ and $A>T$ in trans.

As noted with a dagger symbol in table 1, atypical phenotypes were observed in nine patients. Notably, among the four patients carrying two mutations in SLC26A4, one patient was found to have asymmetric hearing loss despite having bilateral Mondini malformation and two patients were found to have bilateral malformations of the semicircular canals. Among the five patients carrying one mutation in SLC26A4, one patient had unilateral hearing loss with unilateral cystic vestibulocochlear anomaly and auditory nerve hypoplasia on the same side as the hearing loss, one patient had unilateral hearing loss and a unilateral enlarged vestibular aqueduct on the same side as the hearing loss, and one patient also carried a novel DNA sequence VUS and had asymmetric hearing loss despite having bilateral Mondini malformation. Among the two patients carrying one or more VUS in SLC26A4, one patient carrying two VUS had unilateral hearing loss despite having bilateral inner ear malformations involving the vestibule and semicircular canals, and one patient carrying a single VUS had bilateral Mondini malformation with bilateral semicircular canal abnormalities and multiple congenital anomalies of unknown but presumably independent aetiology. Additionally, one patient with a clinical diagnosis of Usher syndrome type 1 carrying three apparently homozygous VUS in $\mathrm{CDH} 23$ had bilateral malformation of the semicircular canals.

\section{DISCUSSION}

Briefly, 37 different DNA sequence variations of known or uncertain pathogenicity were identified in the coding and near-coding regions of 9 genes in 25 of 71 patients with hearing loss whose clinical presentation suggested a genetic syndrome or auditory neuropathy. Of these DNA sequence variations, 14 were understood to be novel at the time this manuscript was written. Atypical phenotypes were observed in 9 patients.

Eight patients with one or more DNA sequence variations in SLC26A4 and one patient with DNA sequence variations in $\mathrm{CDH} 23$ demonstrated additional physical findings not typically thought of as associated with mutations in these genes. In three of these patients, two mutations in SLC26A4 were identified. In six patients, five with variations in SLC26A4 and one with variations in $\mathrm{CDH} 23$, only one mutation or one or more VUS were identified. These observations suggest several possibilities: the phenotypic spectrum associated with mutation in these genes may be broader than typically considered; additional mutations in these genes not detected by the methods used in this study might exist in these patients; mutations in causative or modifier genes not evaluated in this study may be involved; or, environmental factors that modify the phenotypes associated with mutations in these genes might exist.

A definite or presumptive molecular aetiology was identified for only 9 of the 71 patients evaluated in this study - 8 suspected of having syndromic hearing loss and 1 with auditory neuropathy. While more extensive sequencing of the regulatory and deep intronic regions of the genes studied might have yielded additional molecular information, the possibility of DNA sequence variations in additional genes or copy number variations must also be considered. Such ambiguities highlight the limitations of traditional gene sequencing approaches that examine only coding and near-coding regions of known causative genes. In contrast, newer technologies 


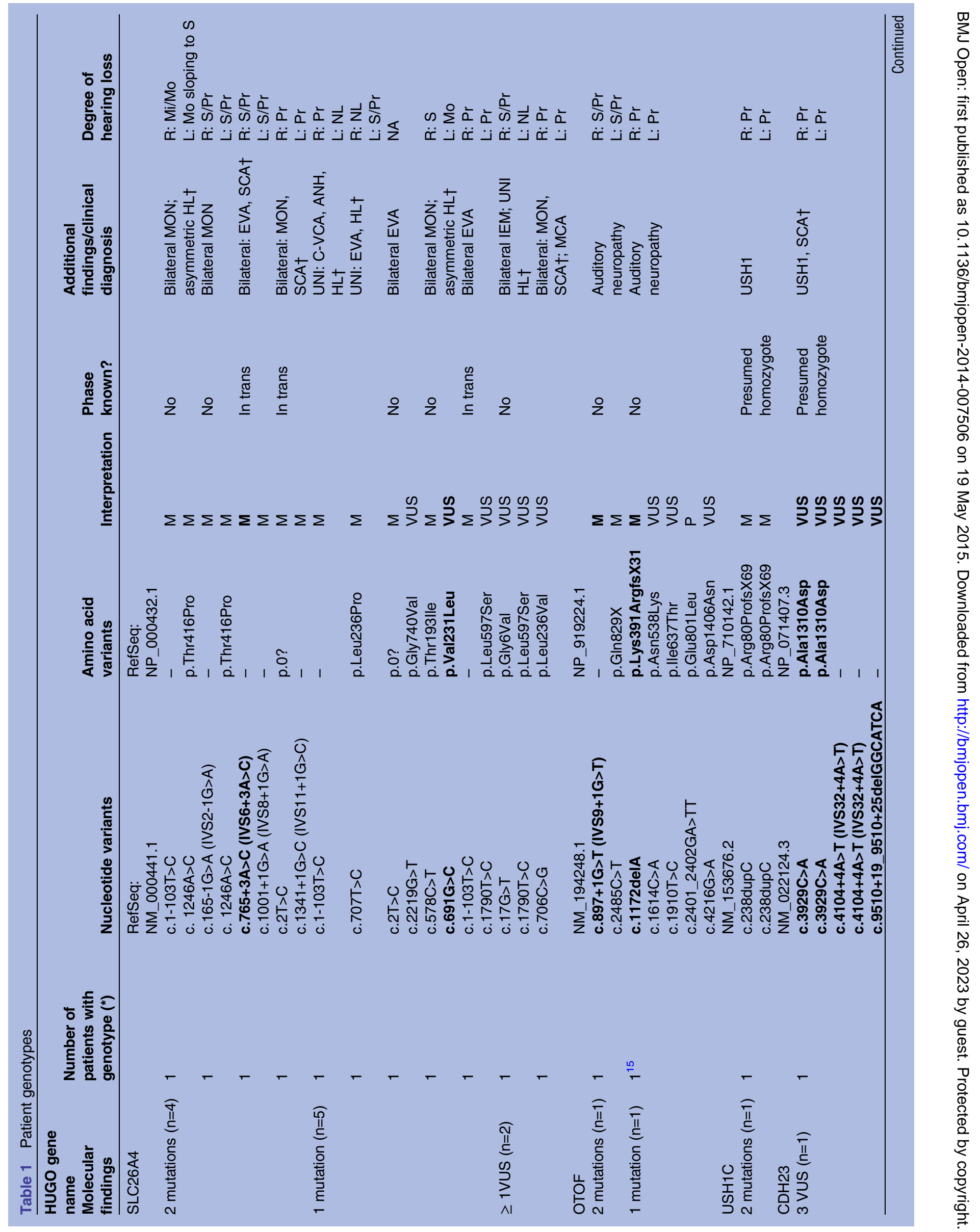




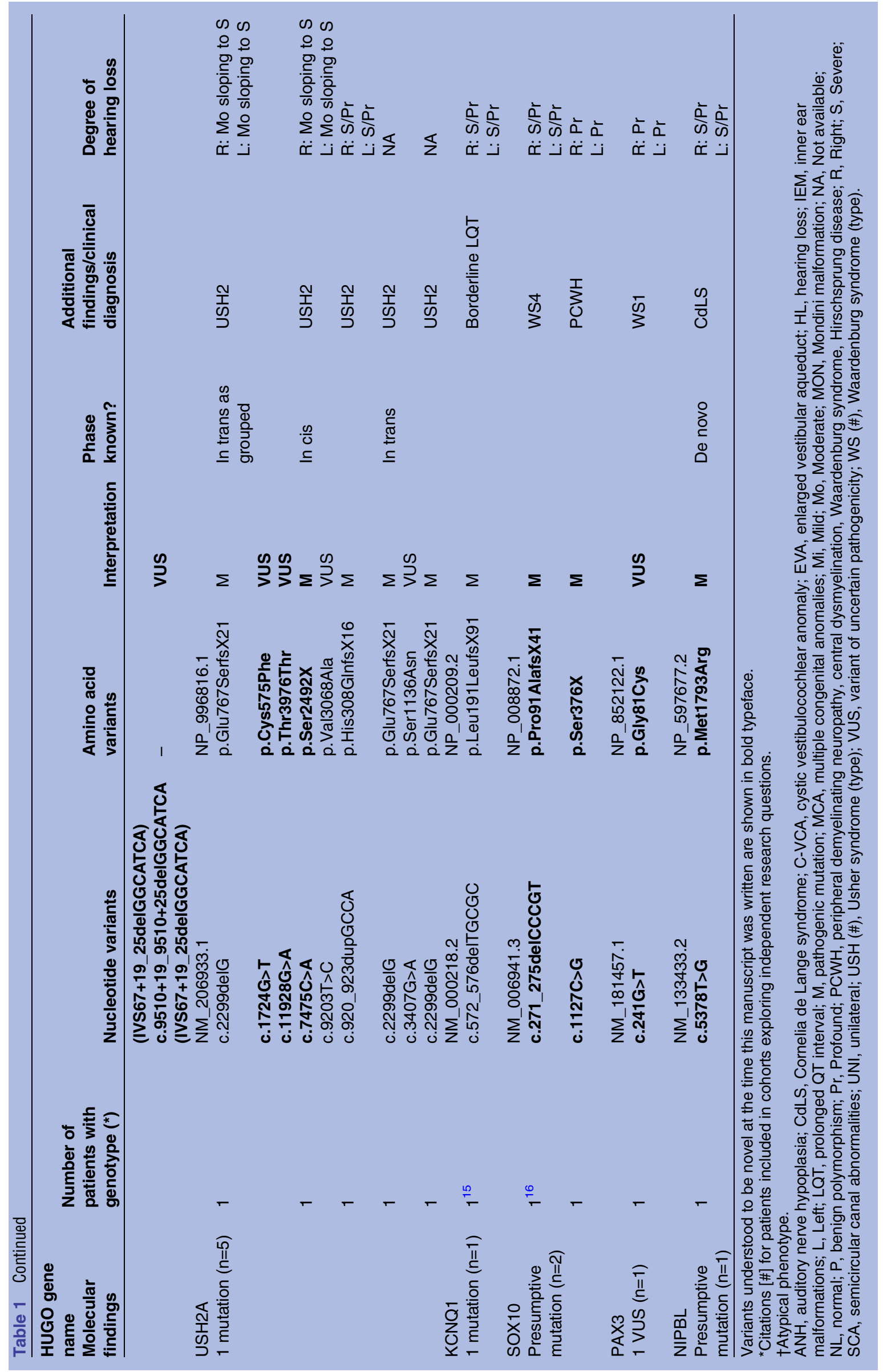


such as whole exome and whole genome sequencing, known as next-generation sequencing technologies, allow sequencing of many genes in a single test and, in the case of whole genome sequencing, permit evaluation of non-coding regions. The more comprehensive genomic coverage of these next-generation sequencing technologies support their consideration for the evaluation of patients with highly heterogeneous conditions such as genetic hearing loss. ${ }^{17-19}$

This study illustrates the clinical utility of DNA sequencing in patients whose presentation suggests a genetic syndrome or auditory neuropathy. The failure to identify a genetic aetiology in many patients in this study highlights the extreme heterogeneity of genetic hearing loss, the incompleteness of current knowledge of aetiologies of hearing loss, and the limitations of conventional DNA sequencing strategies that evaluate only coding and near-coding segments of genes.

\section{Author affiliations}

${ }^{1}$ Bobby R Alford Department of Otolaryngology — Head and Neck Surgery, Baylor College of Medicine, Houston, Texas, USA

${ }^{2}$ Medical Genetics Laboratories, Baylor College of Medicine, Houston, Texas, USA

${ }^{3}$ Department of Molecular and Human Genetics, Baylor College of Medicine, Houston, Texas, USA

${ }^{4}$ Department of Otorhinolaryngology-Head \& Neck Surgery, Albert Einstein College of Medicine of Yeshiva University, Bronx, New York, USA

${ }^{5}$ Myriad Genetic Laboratories, Salt Lake City, Utah, USA

${ }^{6}$ Department of Otolaryngology-Head and Neck Surgery, Stanford University, Stanford, California, USA

Acknowledgements The authors would like to acknowledge the efforts of $\mathrm{Dr}$ Reid Sutton, Dr Carlos Bacino, Dr Fernando Scaglia, Dr Ross Tonini, Claudia Emery and Janet Maxian.

Contributors H-YT, PF, JWL, SD, BBR, JSO, RLA and SM were involved in substantial contributions to conception and design, acquisition of data, and analysis and interpretation of data; drafting the article or revising it critically for important intellectual content; final approval of the version to be published. Agreement to be accountable for all aspects of the work in ensuring that questions related to the accuracy or integrity of any part of the work are appropriately investigated and resolved. BTO and JAH were involved in substantial contributions to acquisition of data, and analysis and interpretation of data, drafting the article or revising it critically for important intellectual content, final approval of the version to be published. Agreement to be accountable for all aspects of the work in ensuring that questions related to the accuracy or integrity of any part of the work are appropriately investigated and resolved. No one who fulfils the criteria for authorship has been excluded as an author.

Funding This work was supported by the Alkek Foundation for Research, Sid Richardson Foundation, Brown Foundation Research and Bintliff Foundation for Research.

Competing interests None declared.

Ethics approval This research was approved by the Institutional Review Board (IRB) of Baylor College of Medicine. A Federal Certificate of Confidentiality was obtained.

Provenance and peer review Not commissioned; externally peer reviewed.

Data sharing statement The informed consent document for this study was developed prior to contemporary data sharing prescriptions. In compliance with the informed consent document for this study, all relevant data is provided in this article.

Open Access This is an Open Access article distributed in accordance with the Creative Commons Attribution Non Commercial (CC BY-NC 4.0) license, which permits others to distribute, remix, adapt, build upon this work noncommercially, and license their derivative works on different terms, provided the original work is properly cited and the use is non-commercial. See: http:// creativecommons.org/licenses/by-nc/4.0/

\section{REFERENCES}

1. Keats BJ, Berlin Cl. Genomics and hearing impairment. Genome Res 1999:9:7-16.

2. Petit C, Levilliers J, Hardelin JP. Molecular genetics of hearing loss. Annu Rev Genet 2001;35:589-646.

3. Toriello H, Smith S. Hereditary hearing loss and its syndromes. 3rd edn. New York: Oxford University Press, 2013.

4. Van Camp G, Smith RJH. Hereditary hearing loss homepage. http:// dnalab-www.uia.ac.be/dnalab/hhh/; http://hereditaryhearingloss.org/ (accessed 19 Mar 2015).

5. Smith RJH, Gurrola JG II, Kelley PM. OTOF-Related deafness. 29 Feb 2008 [Updated 14 Jun 2011]. In: Pagon RA, Adam MP Ardinger $\mathrm{HH}$, et al, eds. GeneReviews $\AA$. Seattle, WA: University of Washington, Seattle, 1993-2015. http://www.ncbi.nlm.nih.gov/books/ NBK1251/ (accessed 19 Mar 2015).

6. Lin JW, Chowdhury N, Mody A, et al. Comprehensive diagnostic battery for evaluating sensorineural hearing loss in children. Otol Neurotol 2011;32:259-64.

7. Ogino S, Gulley ML, den Dunnen JT, et al. Standard mutation nomenclature in molecular diagnostics: practical and educational challenges. J Mol Diagn 2007;9:1-6.

8. Baux D, Faugere V, Larrieu L, et al. UMD-USHbases: a comprehensive set of databases to record and analyse pathogenic mutations and unclassified variants in seven Usher syndrome causing genes. Hum Mutat 2008;29:E76-87.

9. Pendred/BOR Homepage. Molecular otolaryngology research laboratory. lowa City, IA: University of lowa Hospitals and Clinics, Department of Otolaryngology-Head and Neck Surgery. http:// www.healthcare.uiowa.edu/labs/pendredandbor/index.htm (accessed 11 Nov 2014).

10. Sherry ST, Ward MH, Kholodov M, et al. dbSNP: the NCBI database of genetic variation. Nucleic Acids Res 2001;29:308-11.

11. The Human Gene Mutation Database. The Human Gene Mutation Database. Institute of Medical Genetics in Cardiff, Cardiff University. http://www.hgmd.cf.ac.uk/ac/index.php (accessed 11 Nov 2014).

12. Adzhubei IA, Schmidt S, Peshkin L, et al. A method and server for predicting damaging missense mutations. Nat Methods 2010;7:248-9.

13. $\mathrm{Ng} \mathrm{PC}$, Henikoff S. Predicting deleterious amino acid substitutions. Genome Res 2001;11:863-74

14. ACMG Laboratory Practice Committee Working Group. ACMG recommendations for standards for interpretation of sequence variations. Genet Med 2000;2:302-3

15. Tang HY, Xia A, Oghalai JS, et al. High frequency of the IVS2$2 A>G$ DNA sequence variation in SLC26A5, encoding the cochlear motor protein prestin, precludes its involvement in hereditary hearing loss. BMC Med Genet 2005:6:30.

16. Tang HY, Fang P, Ward PA, et al. DNA sequence analysis of GJB2, encoding connexin 26: observations from a population of hearing impaired cases and variable carrier rates, complex genotypes, and ethnic stratification of alleles among controls. Am J Med Genet $A$ 2006;140:2401-15.

17. Brownstein $\mathrm{Z}$, Friedman LM, Shahin $\mathrm{H}$, et al. Targeted genomic capture and massively parallel sequencing to identify genes for hereditary hearing loss in Middle Eastern families. Genome Biol 2011;12:R89.

18. Shearer AE, Deluca AP, Hildebrand MS, et al. Comprehensive genetic testing for hereditary hearing loss using massively parallel sequencing. Proc Natl Acad Sci USA 2010;107:21104-9.

19. Vona B, Muller T, Nanda I, et al. Targeted next-generation sequencing of deafness genes in hearing-impaired individuals uncovers informative mutations. Genet Med 2014;16:945-53. 